02

\title{
Динамика энергообмена и релаксация возбуждений при сильном экситон-плазмонном взаимодействии в планарной наноструктуре из молекулярных $J$-агрегатов на металлической подложке
}

\author{
(С) М.Г. Кучеренко, Т.М. Чмерева \\ Оренбургский государственный университет, \\ Центр лазерной и информационной биофизики, \\ 460018 Оренбург, Россия \\ e-mail: rphys@mail.osu.ru
}

Поступила в редакцию 30.09.2016 г.

В окончательной редакции 03.04.2018 г.

\begin{abstract}
Исследована кинетика энергообмена между плазмонной и экситонной подсистемами в планарной композитной наноструктуре „металл-диэлектрик-молекулярный слой“ при сильном экситон-плазмонном взаимодействии. Показано, что временная зависимость передачи энергии между компонентами системы имеет характер затухающих осцилляций, зависящих от приготовленного начального состояния, ряда релаксационных параметров, частоты Раби и отстройки от резонанса.
\end{abstract}

DOI: $10.21883 /$ OS.2018.08.46354.310-17

Активация коллективных электронных возбуждений - экситонов в кристаллических полупроводниках (диэлектриках) и плазмонов в металлах, определяет многие физические свойства как самих конденсированных систем, так и их композиций, включая и особенности их взаимодействия с внешним электромагнитным полем [1-14]. В гибридных наноструктурах „металл-полупроводник“, „металл-диэлектрик“ или „полупроводник-диэлектрик“ имеют место экситон-плазмонное [3-11] или межвидовое экситонэкситонное взаимодействия [12-15], которые вносят свой вклад в формирование электронных и оптических характеристик многокомпонентных систем. Как в экспериментальных [4-6], так и теоретических [7-9] работах была показана возможность возникновения гибридных поверхностных плазмон-экситонных состояний в композитных наноструктурах. Авторами этих работ установлено, что в зависимости от состава и строения наносистем энергия взаимодействия между экситонным состоянием и плазмонной модой (расщепление Раби) в условиях точного резонанса может доходить до полутора сотен миллиэлектрон-вольт.

В [15-20] рассмотрены различные модели безызлучательной передачи энергии с участием экситонов [15-16], или поверхностных плазмонов [17-19], вблизи плоских границ раздела сред $[15,20]$, нанопроволок $[16,17]$, либо сферических наночастиц $[18,19]$.

В [21] теоретически исследована трансформация энергии электронного возбуждения в планарной „сэндвичевой“" наноструктуре, состоящей из металлической подложки, диэлектрической прослойки и двумерного монослоя $J$-агрегатов цианиноваго красителя. В рамках квантово-механической теории возмущений были проведены расчеты скорости передачи энергии от поверх- ностных плазмонов, возбуждаемых в подложке, например, электронами, к $J$-агрегатам в условиях слабого экситон-плазмонного взаимодействия. Было показано, что для определенных значений параметров системы скорость радиационного распада двумерного френкелевского экситона становится выше скорости тушения экситонного состояния металлом. Это обстоятельство позволяет считать перспективным использование таких слоистых структур в светоизлучающих устройствах нового поколения [4,5]. Кроме того, в [21] установлена зависимость энергии плазмон-экситонного взаимодействия от параметров системы и рассмотрено гибридное экситон-плазмонное состояние. Образование такого состояния возможно, если взаимодействие поверхностных плазмон-поляритонов с экситонами двумерного слоя $J$-агрегатов превышает взаимодействия других типов, которые определяют механизмы релаксации электронных возбуждений в системе, и имеет место пересечение дисперсионных кривых экситонов и плазмонполяритонов.

Если оператор Гамильтона не взаимодействующих друг с другом плазмонов и экситонов имеет два близких собственных значения $E_{\mathrm{pl}}=\hbar \omega(k)$ и $E_{\text {exc }}(\mathbf{k})$, которым соответствуют собственные состояния $|1\rangle=\left|0_{\mathrm{exc}}, 1_{\mathrm{pl}}\right\rangle$ (без экситона, но с одним плазмоном) и $|2\rangle=\left|1_{\mathrm{exc}}, 0_{\mathrm{pl}}\right\rangle$ (с одним экситоном в отсутствие плазмона) объединенной системы, а все остальные собственные значения расположены далеко, то при вычислении поправок целесообразно уже в нулевом приближении использовать суперпозицию $| \pm\rangle=\alpha|1\rangle \pm \beta|2\rangle$ [7,22]. Тогда из условия разрешимости системы уравнений для коэффициентов $\alpha, \beta$ суперпозиции следуют два значения энергии состояний $|+\rangle$ и $|-\rangle$ плазмон-экситонного гибрида 


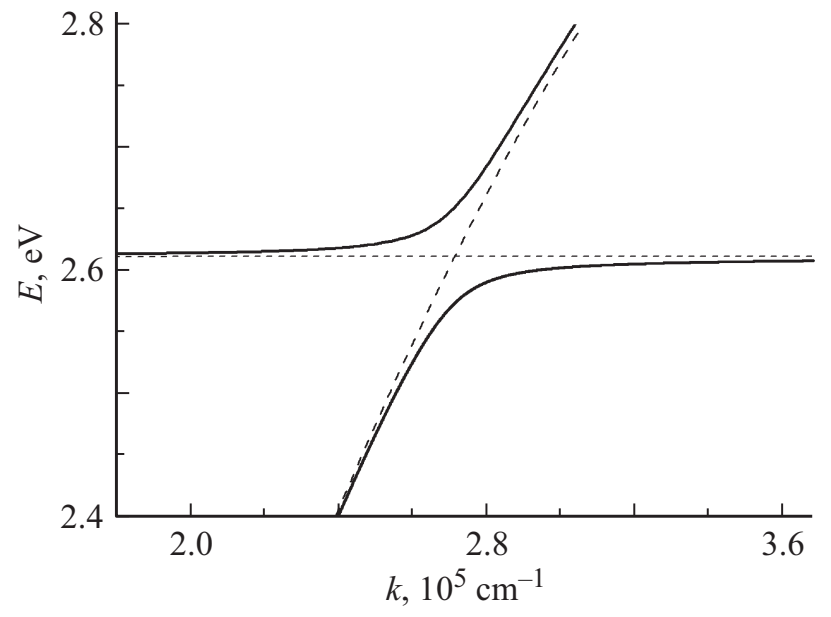

Рис. 1. Дисперсионные кривые гибридной экситон-плазмонной системы. Резонансная область наиболее выраженного взаимодействия состояний приходится на небольшой диапазон волновых чисел: от $2.5 \cdot 10^{5}$ до $3 \cdot 10^{5} \mathrm{~cm}^{-1}$.

(рис. 1):

$$
\begin{aligned}
E_{ \pm}(\mathbf{k})= & \frac{1}{2}\left(E_{\mathrm{exc}}(\mathbf{k})+\hbar \omega(k)\right) \\
& \pm \frac{1}{2} \sqrt{\left(E_{\mathrm{exc}}(\mathbf{k})-\hbar \omega(k)\right)^{2}+4\left|V_{10,01}(\mathbf{k})\right|^{2}}
\end{aligned}
$$

где $E_{\mathrm{exc}}(\mathbf{k})-$ энергия двумерного экситона, $\hbar \omega(k)$ - энергия поверхностного плазмон-поляритона, $V_{10,-1}(\mathbf{k})$ - матричный элемент экситон-плазмонного взаимодействия $[21], \mathbf{k}-$ волновой вектор гибридной квазичастицы, $k=|\mathbf{k}|$. Для матричного элемента $\langle 1|V| 2\rangle=V_{10,01}(\mathbf{k})$ экситон-плазмонного взаимодействия была установлена следующая зависимость его значения от толщины $l$ изолирующей прослойки с диэлектрической проницаемостью $\varepsilon_{1}$ между металлом и молекулярным $J$-слоем:

$$
V_{10,01}(l) \sim\left[\operatorname{ch}\left(\kappa_{z}^{(1)} l\right)+\frac{\varepsilon_{2} \kappa_{z}^{(1)}}{\varepsilon_{1} \kappa_{z}^{(2)}} \operatorname{sh}\left(\kappa_{z}^{(1)} l\right),\right]
$$

для случая, когда монослой $J$-агрегатов находится в непроводящей среде с диэлектрической проницаемостью $\varepsilon_{2}$. Действительные показатели $k_{z}^{1(2)}=$ $=\sqrt{k^{2}-\varepsilon_{1(2)} \omega^{2} / c^{2}}$ в (2) характеризуют быстроту спадания амплитуды ближнего поля вдоль внешней нормали $z$ к поверхности металла, $c$ - скорость света. Таким образом, как видно из (2), при достаточно больших значениях толщины прослойки экситон-плазмонное взаимодействие экспоненциально убывает с ростом $l$. Изменяя толщину $l$, можно регулировать константу связи $\langle 1|V| 2\rangle$, осуществляя плавный переход от сильного взаимодействия к слабому.

Как отмечалось в [21], в случае сильного экситонплазмон-поляритонного взаимодействия, которое для рассмотренной системы может достигать величины $\sim 100 \mathrm{meV}$, необходимо производить описание трансформации энергии на основе квантово-механического формализма [23], использующего парную матрицу плотности $\rho_{i j}$ квантовых подсистем, взаимодействующих как друг с другом, так и с термостатом [24,25].

\section{Модель кинетики энергообмена при сильном взаимодействии компонентов}

Оператор плотности $\widehat{\rho}(t)$ объединенной системы удовлетворяет кинетическому уравнению, записанному на базе уравнения Неймана с релаксационным слагаемым

$$
\frac{d}{d t} \widehat{\rho}(t)=\frac{1}{i \hbar}\left[\widehat{H}_{0}+\widehat{V}, \widehat{\rho}\right]-\widehat{R}^{\dagger} \widehat{\rho}
$$

Оператор $\widehat{H}_{0}$ в $(3)$ - гамильтониан объединенной системы в отсутствие экситон-плазмонного взаимодействия, $\widehat{R}^{\dagger}$-супероператор релаксации [25].

В простейшей релаксационной модели вводятся времена $\tau_{1,2}$ релаксации населенности состояний $|1\rangle$ и $|2\rangle$ и время $T_{2}$ фазовой релаксации - затухания недиагональных элементов матрицы плотности. В этом случае элементы матрицы плотности удовлетворяют следующей системе четырех дифференциальных уравнений [24]:

$$
\begin{aligned}
& \frac{d}{d t}\left(\begin{array}{l}
\rho_{11} \\
\rho_{22} \\
\rho_{12} \\
\rho_{21}
\end{array}\right) \\
& =\left(\begin{array}{cccc}
-\frac{1}{\tau_{\mathrm{pl}}} & 0 & i \frac{V_{21}}{\hbar} & -i \frac{V_{12}}{\hbar} \\
0 & -\frac{1}{\tau_{\mathrm{exc}}} & -i \frac{V_{21}}{\hbar} & i \frac{V_{12}}{\hbar} \\
i \frac{V_{12}}{\hbar} & -i \frac{V_{12}}{\hbar} & -\frac{1}{T_{2}}-i \frac{\Delta E}{\hbar} & 0 \\
-i \frac{V_{21}}{\hbar} & i \frac{V_{21}}{\hbar} & 0 & -\frac{1}{T_{2}}+i \frac{\Delta E}{\hbar}
\end{array}\right)\left(\begin{array}{l}
\rho_{11} \\
\rho_{22} \\
\rho_{12} \\
\rho_{21}
\end{array}\right),
\end{aligned}
$$

или коротко

$$
\dot{X}=A X(t) \text {. }
$$

Здесь $\tau_{\mathrm{pl}}=\tau_{1}$ и $\tau_{\mathrm{exc}}=\tau_{2}-$ времена жизни плазмона и экситона, $V_{12}=\langle 1|\widehat{V}| 2\rangle, \Delta E=\left\langle 2\left|\widehat{H}_{0}\right| 2\right\rangle-\left\langle 1\left|\widehat{H}_{0}\right| 1\right\rangle$.

Формальное решение уравнения (5) в виде $X=$ $=\exp (A t) X(0)$ может быть получено с помощью известной теоремы Сильвестра [26]

$$
\exp (A t)=\sum_{k=1}^{n} \exp \left(\lambda_{k} t\right)\left[\prod_{j \neq k}\left(\lambda_{k}-\lambda_{j}\right)\right]^{-1} \prod_{j \neq k}\left(A-\lambda_{j} I\right)
$$

где собственные значения $\lambda_{j}$ матрицы $A$ определяются из уравнения четвертого порядка $\operatorname{det}[A-\lambda I]=0$. 
Решения системы уравнений (4), (5) для некоторых частных случаев совпадающих времен релаксации анализировались и ранее [23,27]. Однако, как справедливо отмечено авторами [18], детальный анализ общего случая должен представлять собой предмет отдельного исследования.

В случае, когда $\tau_{\mathrm{pl}}=\tau_{\mathrm{exc}}=\tau$ и в условиях точного резонанса $\Delta E=0$ собственные значения $\lambda_{j}$ матрицы $A$ принимают вид

$$
\begin{gathered}
\lambda_{1,2}=-\frac{1}{2}\left[\left(1 / \tau+1 / T_{2}\right) \pm \sqrt{\left(1 / \tau-1 / T_{2}\right)^{2}-4 \Omega^{2}}\right] \\
\lambda_{3}=-1 / \tau \quad \lambda_{4}=-1 / T_{2}, \quad \Omega=2\left|V_{12}\right| / \hbar .
\end{gathered}
$$

Как известно, характер осцилляций Раби сильно зависит от начальных условий, в качестве которых выберем, например, $\rho_{11}(0)=1, \rho_{22}=(0)=0$, что отвечает начальной активации лишь плазмонной подсистемы. Обсуждение конкретной экспериментальной ситуации, отвечающей реализации такого начального условия, проведем ниже, а пока запишем простые выражения для населенностей $\rho_{11}(t)=\rho^{(+)}, \rho_{22}(t)=\rho^{(-)}$состояний $|1\rangle$ и $|2\rangle$ (здесь обозначения „+,“ и „-““ не связаны с гибридными состояниями для энергий (1)), при стартовом заселении $\rho_{11}(0)=1$ состояния $|1\rangle$ :

$\rho^{( \pm)}(t)=\frac{1}{2}\left[\exp \left(-\frac{t}{\tau}\right) \pm C \exp \left(\lambda_{1} t\right) \pm(1-C) \exp \left(\lambda_{2} t\right)\right]$,

где

$$
\begin{gathered}
C=\frac{\left(1 / \tau-1 / T_{2}\right)+\sqrt{\left(1 / \tau-1 / T_{2}\right)^{2}-4 \Omega^{2}}}{2 \sqrt{\left(1 / \tau-1 / T_{2}\right)^{2}-4 \Omega^{2}}}, \\
\Omega=2\left|V_{12}\right| / \hbar .
\end{gathered}
$$

Переключение кинетики населенностей с чисто релаксационного на осцилляционно-релаксационный режим происходит по достижению критического значения параметров $1 / \tau_{\mathrm{exc}}-1 / T_{2}=4\left|V_{12}\right| / \hbar$, когда собственные значения $\lambda_{j}$ матрицы $A$ становятся комплексными. Заметим, что из (7) тогда получаем $\lambda_{1}=\lambda_{2}^{*}$ при $2 \Omega>1 / \tau_{\mathrm{exc}}-1 / T_{2}$, а из (9) $1-C=C^{*}$. Таким образом, и в случае комплексных значений $\lambda_{j}$, населенности $\rho^{(+)}, \rho^{(-)}$определяемые (8), остаются действительными величинами, и могут быть записаны в виде

$$
\begin{gathered}
\rho^{( \pm)}(t)=\frac{1}{2}\left[\exp \left(-\frac{t}{\tau} \pm 2 \operatorname{Re}\left(C \exp \left(\lambda_{1} t\right)\right)\right)\right], \\
C=\frac{1}{2} \frac{\left(1 / \tau-1 / T_{2}\right)+i \sqrt{4 \Omega^{2}-\left(1 / \tau-1 / T_{2}\right)^{2}}}{i \sqrt{4 \Omega^{2}-\left(1 / \tau-1 / T_{2}\right)^{2}}} .
\end{gathered}
$$

Отметим также, что при равенстве времен жизни экситона и плазмона $\tau_{\mathrm{pl}}=\tau_{\mathrm{exc}}=\tau$, произвольном времени $T_{2}$ фазовой релаксации и нулевой отстройке от резонанса, $\Delta E=0$, на основе системы (4) методом исключения переменных можно получить автономные уравнения для инверсии $\Delta n(t)=\rho_{11}(t)-\rho_{22}(t)$ и суммарной населенности $n(t)=\rho_{11}(t)+\rho_{22}(t)$ возбужденного состояния системы:

$$
\begin{gathered}
\Delta \ddot{n}(t)+\left(\frac{1}{\tau}+\frac{1}{T_{2}}\right) \Delta \dot{n}(t)+\left(\Omega^{2}+\frac{1}{\tau T_{2}}\right) \Delta n(t)=0, \\
\dot{n}(t)=-n(t) / \tau .
\end{gathered}
$$

Учитывая, что $\Delta n(t)+n(t)=2 \rho_{11}(t)$ и $n(t)-\Delta n(t)=$ $=2 \rho_{22}(t)$, можем легко определить кинетику населенностей $\rho_{11}(t), \rho_{22}(t)$ после нахождения решений уравнений (12). Отметим, что собственные числа уравнения (12) совпадают с собственными значениями $\lambda_{1,2}$, определенными выражением (7).

При сильном экситон-плазмонном взаимодействии, когда частота Раби $\Omega=2\left|V_{12}\right| / \hbar \gg 1 / \tau_{\text {exc }}, 1 / T_{2}$, на временах $t \sim \Omega^{-1} \ll \tau_{\text {exc }}, T_{2}$ из (11) получаем для инверсии $\Delta n(t)$ уравнение гармонических колебаний с частотой $\Omega$ :

$$
\Delta \ddot{n}(t)+\Omega^{2} \Delta n(t)=0,
$$

откуда $\Delta n(t)=\cos (\Omega t)$. В этих условиях из второго уравнения $(12)$ следует $n(t) \approx 1$, и тогда

$$
\begin{aligned}
& \rho_{11}(t)=[1+\cos (\Omega t)] / 2, \\
& \rho_{22}(t)=[1-\cos (\Omega t)] / 2 .
\end{aligned}
$$

То есть на временах $t \sim \Omega^{-1} \ll \tau_{\text {ехс }}, T_{2}$ существенно меньших всех времен релаксации системы, между экситонами и плазмонами планарной наноструктуры успевает произойти многократный взаимный энергообмен с частотой Раби $\Omega$. Гармонические осцилляции населенностей (13) будут медленно затухать по единому экспоненциальному закону со скоростью $1 / \tau$. В случае $\tau_{\mathrm{pl}}=\tau_{\mathrm{exc}}=T_{2}$ и в условиях точного резонанса, $\Delta E=0$, спектр собственных значений $\lambda_{j}$ матрицы $A$ становится вырожденным:

$$
\lambda_{1}=\lambda_{2}=-1 / \tau, \quad \lambda_{3}=\lambda_{4}^{*}=-1 / \tau-i \Omega .
$$

В случае произвольных значений $\Omega, 1 / \tau, 1 / T_{2}$ на основе (10) и (11) получаем кинетику (8), качественно отраженную на рис. $2, a-c$ и $3, a-b$. Постоянная $C$ в (8) может быть определена на основе первого уравнения системы (4) при $t \rightarrow 0: \dot{\rho}_{11}=-1 / \tau$. Тогда

$$
\begin{gathered}
C=\frac{1}{2}\left[1+\frac{1}{\sqrt{1-\eta^{2}}}\right], \\
1-C=\frac{1}{2}\left[1-\frac{1}{\sqrt{1-\eta^{2}}}\right], \quad \eta=\frac{2 \Omega}{\left(1 / \tau-1 / T_{2}\right)},
\end{gathered}
$$

что, конечно, совпадает с (9).

Обсудим теперь, каким образом может быть практически реализована экспериментальная ситуация, отвечающая выбору начального условия в виде парциальной активации плазмонной подсистемы. 

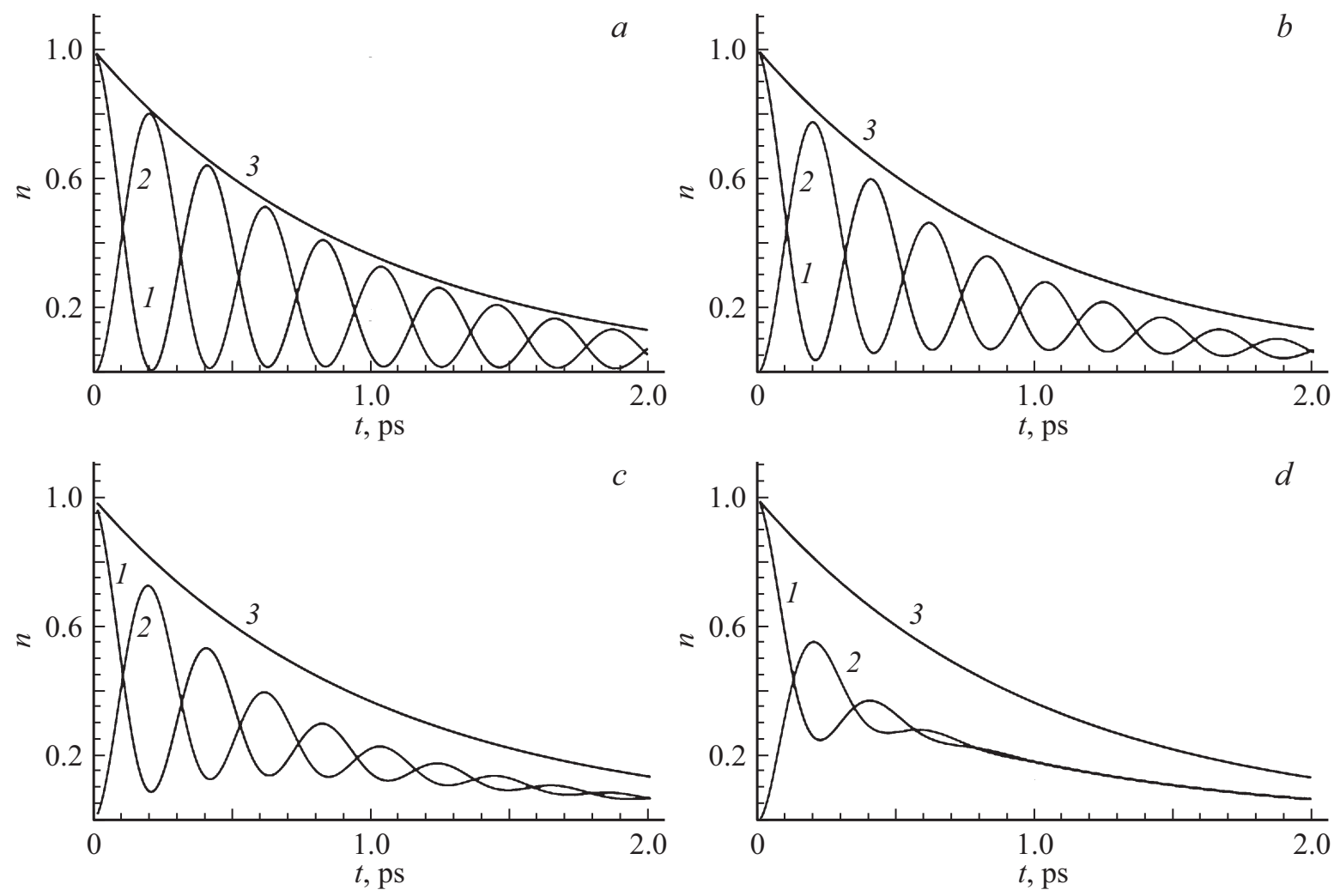

Рис. 2. Кинетика обмена-релаксации населенностей плазмонной $\rho_{11}(t)(1)$ и экситонной $\rho_{22}(t)(2)$ подсистем, а также суммарной населенности $n(t)=\rho_{11}+\rho_{22}(3)$ в интерактивной плазмон-экситонной системе при начальной активации плазмонной компоненты, $\tau_{\mathrm{pl}}=\tau_{\mathrm{exc}} \sim 10^{-12} \mathrm{~s}$, частоте Раби $\Omega=15 / \tau_{\text {exс }}$ и различных временах фазовой релаксации: $T_{2}=0.8 \tau_{\mathrm{exc}}(a), 0.5 \tau_{\mathrm{exc}}(b), 0.3 \tau_{\mathrm{exc}}(c)$, $0.1 \tau_{\mathrm{exc}}(d)$. Время $t$ выражено в рs. Точная настройка на резонанс $\Delta E=0$.
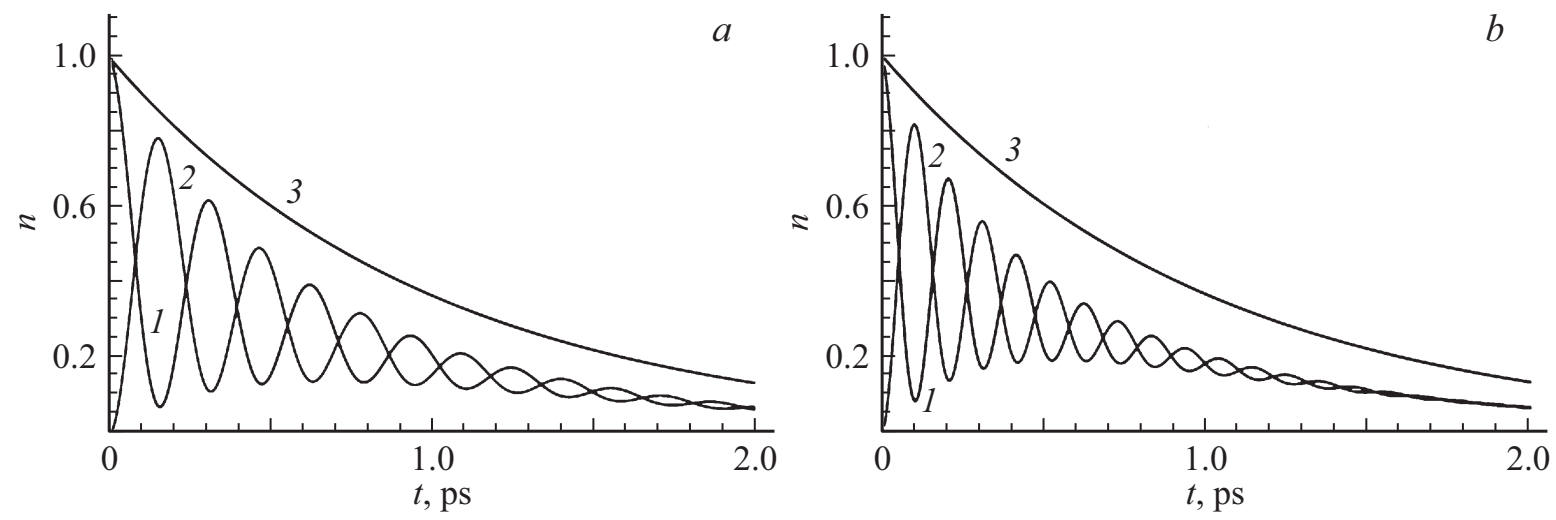

Рис. 3. Кинетика обмена-релаксации населенностей плазмонной $\rho_{11}(t)(1)$ и экситонной $\rho_{22}(t)(2)$ подсистем, а также суммарной населенности $n(t)=\rho_{11}+\rho_{22}(3)$ в интерактивной плазмон-экситонной системе при начальной активации плазмонной компоненты, различных временах фазовой релаксации $T_{2}=0.3 \tau_{\mathrm{exc}}(a), 0.2 \tau_{\mathrm{exc}}(b)$ и частотах Раби $\Omega=20 / \tau_{\mathrm{exc}}(a), 30 / \tau_{\mathrm{exc}}(b) . \tau_{\mathrm{pl}}=\tau_{\mathrm{exc}} \sim 10^{-12} \mathrm{~s}$. Время $t$ выражено в рs. Точная настройка на резонанс $\Delta E=0$.

В общем случае стационарные состояния гибридной экситон-плазмонной системы представляют собой суперпозицию чисто плазмонных и чисто экситонных состояний. Приготовление состояния, в котором активирована только плазмонная мода $|1\rangle$, является созданием нестационарного состояния, из которого возможен переход в другое нестационарное состояние $|2\rangle$ с активизацией только экситонной моды. Формирование парциального плазмонного возбуждения может быть произведено экспериментально одним из следующих способов.

Импульсно-энергетическая схема приготовления начального состояния.

Активация только плазмонной моды с фиксированным волновым вектором $\mathbf{k}$ может быть осуществлена в диа- 
пазоне волновых чисел вне области пересечения дисперсионных кривых плазмонов и экситонов (например, со значениями $k$, большими резонансных), т. е. вне области формирования гибрида (рис. 1). Такая процедура может быть произведена, например, известным методом с использованием плоской световой волны и специальной призмы в конфигурации Кречмана. Далее в результате межмодового плазмонного взаимодействия будет происходить перекачка энергии из коротковолновых мод в длинноволновые, и как следствие этого - парциальная активация плазмонных мод в резонансном диапазоне значений волновых чисел.

В частности, межмодовый переброс поверхностных плазмонов в область пересечения дисперсионных кривых может быть осуществлен в результате их неупругого рассеяния на поверхностных фононах с частотами $\omega_{\mathrm{Ph}}(\mathbf{k})$ :

$$
\omega(\mathbf{k})=\omega^{\prime}\left(\mathbf{k}^{\prime}\right)+\omega_{\mathrm{Ph}}\left(\mathbf{k}-\mathbf{k}^{\prime}\right) .
$$

Кроме того, в качестве особого способа возбуждения парциальной плазмонной моды гибридной системы можно рассматривать воздействие на поверхность металла электронным пучком. Воздействие на плазму излучения большой мощности изменяют свойства среды и безусловно особым образом влияют на плазмонный межмодовый обмен. Однако мы оставляем здесь без обсуждения экзотические для рассматриваемого случая невысокого уровня накачки нелинейные эффекты взаимодействия коллективных возбуждений (мод) плазмы.

Другим возможным вариантом активации парциального плазмонного начального состояния является пространственно-временная схема его приготовления (рис. 4).

Пространственно-временная схема приготовления начального состояния.

В этом варианте формирования стартового плазмонного состояния производится пространственно селективная активация открытого участка поверхности проводящего слоя под призмой Кречмана, т.е. участка, не покрытого слоем $J$-агрегатов в резонансном диапазоне волновых чисел, отвечающих образованию гибридного состояния. Активированные свободные плазмоны инжектируются затем в область с выраженным экситон-плазмонным взаимодействием, в которой и будет осуществляться энергообмен плазмонной и экситонной подсистем с временными осцилляциями населенностей.

В альтернативном варианте приготовления начальной конфигурации системы может осуществляться непосредственное возбуждение стационарного состояния гибрида в резонансной области волновых чисел. Активация такого начального состояния отвечает одновременному присутствию в нем и плазмонной и экситонной составляющих. В то же время предлагаемая ниже расчетная процедура позволяет определять временную зависимость населенности парциального экситонного

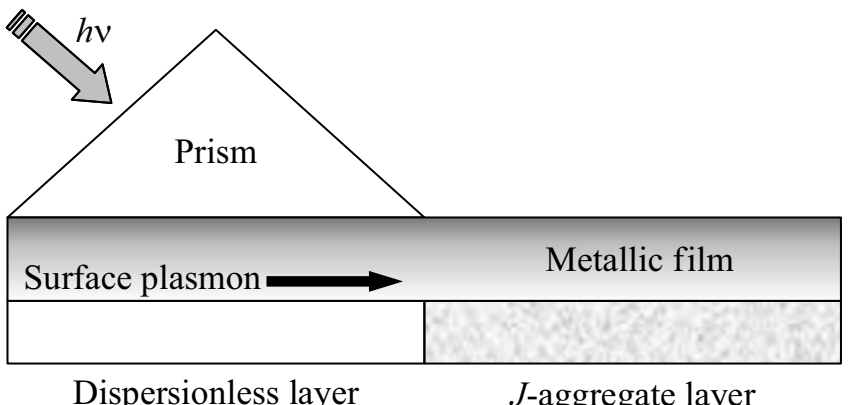

Рис. 4. Пространственно-селективная активация плазмонов на открытом участке поверхности проводящего слоя (без охвата сопряженной экситоногенной области) с помощью призмы Кречмана.

состояния как матричный элемент $\rho_{22}(t)$ оператора плотности $\widehat{\rho}$ для произвольного начального состояния. Тогда величина $\rho_{22}(t)$ определяет вероятность переноса энергии в экситонную моду $|2\rangle$ из суперпозиционного состояния $|+\rangle \rightarrow|2\rangle$ к моменту времени $t$ при реализации произвольной стартовой конфигурации.

Безусловно предложенная импульсно-энергетическая схема приготовления начального состояния является дискуссионной. Более того, в определенной степени дискуссионной является и вторая - пространственновременная схема. В настоящее время обе предложенные схемы носят лишь иллюстративный характер, поскольку пока еще они не реализованы в конкретных экспериментах. Лишь после этого можно будет уверенно говорить о возможности воплощения этих схем.

При произвольных соотношениях между временами $\tau_{\mathrm{pl}}, \tau_{\mathrm{exc}}, T_{2}$ релаксации и ненулевой отстройке $\Delta E$ от резонанса спектр собственных значений $\lambda_{j}$ матрицы $A$ определяется весьма громоздкими выражениями, затрудняющими рассмотрение особенностей действия супероператора релаксации $\widehat{R}^{\dagger}$. Однако в этом общем случае анализ кинетики населенностей состояний $|1\rangle$ и $|2\rangle$, равно как и недиагональных матричных элементов $\rho_{i j}$, может быть произведен на основе модифицированного уравнения Джонсона-Меррифильда [28], предложенного для описания спин-селективной аннигиляции экситонов:

$$
\begin{aligned}
\frac{d}{d t} \widehat{\rho} & =-\frac{i}{\hbar}\left[\left(\widehat{H}_{0}+\widehat{V}\right), \widehat{\rho}\right]-\frac{1}{2} \tilde{k}_{1}\left\{\widehat{P}_{1}, \widehat{\rho}\right\}_{+} \\
& -\frac{1}{2} \tilde{k}_{2}\left\{\widehat{P}_{2}, \widehat{\rho}\right\}_{+}-T_{2}^{-1} \widehat{\rho},
\end{aligned}
$$

где $\quad\left\{\widehat{P}_{j}, \widehat{\rho}\right\}=\widehat{P}_{j} \cdot \widehat{\rho}+\widehat{\rho} \cdot \widehat{P}_{j} \quad-\quad$ антикоммутатор, $\widehat{P}_{j}=|j\rangle\langle j|$ - проекционный оператор на состояние $|j\rangle$, $\tilde{k}_{1}, \tilde{k}_{2}$ - аддитивные добавки к скалярным скоростям дезактивации системы по плазмонному и экситонному каналам распада. 
Решение операторного уравнения (16) можно представить с помощью матричных экспонент [28]:

$$
\begin{array}{r}
\widehat{\rho}(t)=\exp \left(-t / T_{2}\right) \exp \left(\widehat{K}^{(-)} t\right) \widehat{\rho}(0) \exp \left(\widehat{K}^{(+)}(t)\right), \quad(17) \\
K_{i j}^{(\mp)}=\left(\begin{array}{cc}
\mp \frac{i}{\hbar}\left(\hbar \omega(k)+V_{11}\right)-\frac{1}{2} \tilde{k}_{1} & \mp \frac{i}{\hbar} V_{12} \\
\mp \frac{i}{\hbar} V_{12} & \mp \frac{i}{\hbar}\left(E_{\mathrm{exc}}+V_{22}\right)-\frac{1}{2} \tilde{k}_{2}
\end{array}\right)
\end{array}
$$

с собственными значениями матриц (18)

$$
\begin{aligned}
\kappa_{1,2}^{(\mp)} & =(\mp) \frac{i}{2}\left[\omega(k)+E_{\mathrm{exc}} / \hbar\right]-\frac{1}{4}\left(\tilde{k}_{1}+\tilde{k}_{2}\right) \\
& \mp \frac{1}{2}\left\{\left[i(\mp) \Delta E / \hbar+\left(\tilde{k}_{1}-\tilde{k}_{2}\right) / 2\right]^{2}-\Omega^{2}\right\}^{1 / 2} .
\end{aligned}
$$

Заметим, что операторы $\widehat{K}^{(-)}$и $\widehat{K}^{(+)}$комплексно сопряжены, а это означает, что комплексно сопряженными будут и собственные значения $\kappa_{1,2}^{(\mp)}: \kappa_{1,2}^{(-)}=\left(\kappa_{1,2}^{(+)}\right)^{*}$.

С помощью теоремы Сильвестра находим операторное решение уравнения (16):

$$
\begin{aligned}
& \widehat{\rho}(t)=\exp \left(-t / T_{2}\right)\left[\frac{\exp \left(\kappa_{1}^{(-)} t\right) \exp \left(\kappa_{1}^{(+)} t\right)}{\left(\kappa_{1}^{(-)}-\kappa_{2}^{(-)}\right)\left(\kappa_{1}^{(+)}-\kappa_{2}^{(+)}\right)}\right. \\
& \times\left(\widehat{K}^{(-)}-\kappa_{1}^{(-)} \widehat{I}\right) \widehat{\rho}(0)\left(\widehat{K}^{(+)}-\kappa_{1}^{(+)} \widehat{I}\right) \\
& +\frac{\exp \left(\kappa_{2}^{(-)} t\right) \exp \left(\kappa_{2}^{(+)} t\right)}{\left(\kappa_{2}^{(-)}-\kappa_{1}^{(-)}\right)\left(\kappa_{2}^{(+)}-\kappa_{1}^{(+)}\right)}\left(\widehat{K}^{(-)}-\kappa_{2}^{(-)} \widehat{I}\right) \widehat{\rho}(0) \\
& \times\left(\widehat{K}^{(+)}-\kappa_{2}^{(+)} \widehat{I}\right)+\frac{\exp \left(\kappa_{1}^{(-)} t\right) \exp \left(\kappa_{2}^{(+)} t\right)}{\left(\kappa_{1}^{(-)}-\kappa_{2}^{(-)}\right)\left(\kappa_{2}^{(+)}-\kappa_{1}^{(+)}\right)} \\
& \times\left(\widehat{K}^{(-)}-\kappa_{1}^{(-)} \widehat{I}\right) \widehat{\rho}(0)\left(\widehat{K}^{(+)}-\kappa_{2}^{(+)} \widehat{I}\right) \\
& +\frac{\exp \left(\kappa_{2}^{(-)} t\right) \exp \left(\kappa_{1}^{(+)} t\right)}{\left(\kappa_{2}^{(-)}-\kappa_{1}^{(-)}\right)\left(\kappa_{1}^{(+)}-\kappa_{2}^{(+)}\right)}\left(\widehat{K}^{(-)}-\kappa_{2}^{(-)} \widehat{I}\right) \widehat{\rho}(0) \\
& \left.\times\left(\widehat{K}^{(+)}-\kappa_{1}^{(+)} \widehat{I}\right)\right]
\end{aligned}
$$

позволяющее записать выражения для временных зависимостей матричных элементов $\rho_{i j}(t)$ при произвольных стартовых конфигурациях $\rho_{i j}$. Если имеет место селективная начальная активация плазмонной подсистемы $\widehat{\rho}(0)=|1\rangle\langle 1|$, то кинетика населенности плазмонного состояния $|1\rangle=\left|0_{\mathrm{exc}}, 1_{\mathrm{pl}}\right\rangle$ представляется суперпозицией четырех экспонент с комплекснозначными показа- телями:

$$
\begin{aligned}
& \langle 1|\hat{\rho}(t)| 1\rangle=\rho_{11}(t)=\frac{\exp \left(\kappa_{1}^{(-)} t\right)}{\left(\kappa_{1}^{(-)}-\kappa_{2}^{(-)}\right)} \frac{\left(K_{11}^{(-)}-\kappa_{1}^{(-)}\right)}{\left(\kappa_{1}^{(+)}-\kappa_{2}^{(+)}\right)} \\
& \times\left[\left(K_{11}^{(+)}-\kappa_{1}^{(+)}\right) \exp \left(\kappa_{1}^{(+)} t\right)-\left(K_{11}^{(+)}-\kappa_{2}^{(+)}\right) \exp \left(\kappa_{2}^{(+)} t\right)\right] \\
& +\frac{\exp \left(\kappa_{2}^{(-)} t\right)}{\left(\kappa_{1}^{(-)}-\kappa_{2}^{(-)}\right)} \frac{\left(K_{11}^{(-)}-\kappa_{2}^{(-)}\right)}{\left(\kappa_{1}^{(+)}-\kappa_{2}^{(+)}\right)} \\
& \times\left[\left(K_{11}^{(+)}-\kappa_{2}^{(+)}\right) \exp \left(\kappa_{2}^{(+)} t\right)-\left(K_{11}^{(+)}-\kappa_{1}^{(+)}\right) \exp \left(\kappa_{1}^{(+)} t\right)\right] .
\end{aligned}
$$

При тех же начальных условиях кинетика населенности экситонного состояния $|2\rangle=\left|1_{\text {exc }}, 0_{\mathrm{pl}}\right\rangle$ дается выражением

$$
\begin{aligned}
& \rho_{22}(t)=\langle 2|\widehat{\rho}(t)| 2\rangle=\frac{K_{21}^{(-)} K_{12}^{(+)}}{\left(\kappa_{1}^{(-)}-\kappa_{2}^{(-)}\right)\left(\kappa_{1}^{(+)}-\kappa_{2}^{(+)}\right)} \\
& \times\left[\exp \left(\left(\kappa_{1}^{(-)}+\kappa_{1}^{(+)}\right) t\right)+\exp \left(\left(\kappa_{2}^{(-)}+\kappa_{2}^{(+)}\right) t\right)\right. \\
& \left.-\exp \left(\left(\kappa_{1}^{(-)}+\kappa_{2}^{(+)}\right) t\right)-\exp \left(\left(\kappa_{2}^{(-)}+\kappa_{1}^{(+)}\right) t\right)\right]
\end{aligned}
$$

т. е. представляет собой суперпозицию экспонент с одинаковыми по модулю весовыми коэффициентами.

\section{Результаты расчетов и обсуждение}

На основе уравнений (7)-(9), (12) и (17)-(22) были исследованы параметрические зависимости кинетики населенностей $\rho_{11}(t), \rho_{22}(t)$ активированной планарной системы с „экситононосным“ $J$-агрегатным слоем на проводящей подложке. Применимость упрощенной модели (7)-(9) к реальным объектам требует дополнительных обоснований. Так, если условие точного резонанса $\Delta E=0$ выполняется в области пересечения экситонной и плазмонной дисперсионных ветвей (или в области максимального гибридного смешения), то равенство времен $\tau_{\mathrm{pl}}=\tau_{\text {exс }}$ продольной релаксации подсистем выглядит, на первый взгляд, экзотичным. На это указывают типичные данные для этих величин, приведенные в литературе для благородных металлов, а также молекулярных кристаллов и $J$-агрегатных структур. Так, по оценкам [5], для скорости релаксации экситонов в $J$-агрегатных слоях цианиновых красителей справедлива оценка $1 / \tau_{\text {exc }}=1.59 \cdot 10^{13} \mathrm{~s}^{-1}$, тогда как характерное время $\tau_{\mathrm{pl}}$ жизни плазмонов для $\mathrm{Au}$ и $\mathrm{Ag}$ составляет $5 \mathrm{fs}$, что соответствует скорости распада плазмонов $\tau_{\mathrm{pl}}^{-1}=2 \cdot 10^{14} \mathrm{~s}^{-1}$. Таким образом, имеет место не равенство характерных времен $\tau_{\mathrm{pl}}$ и $\tau_{\text {exc }}$, а их отличие на порядок величины. С другой стороны, указанное числовое соотношение между этими параметрами имеет место 
для температур порядка комнатных. В низкотемпературной области и $\tau_{\mathrm{pl}}$ и $\tau_{\text {ехс }}$ существенно возрастают, но с разной скоростью. Температурные изменения коэффициента диссипации экситонов для кристалла антрацена, определенные Бродиным и Марисовой [29] на основе экспериментальных данных по отражению света, охватывают диапазон значений от $10^{10} \mathrm{~s}^{-1}$ при температуре $4 \mathrm{~K}$ до $10^{12} \mathrm{~s}^{-1}$ при $200 \mathrm{~K}$. В то же время температурные изменения частоты электрон-фононных столкновений, вычисленные на основе формулы Блоха-Грюнайзена для беспримесного серебра, указывают на то, что в благородных металлах перепад значений $1 / \tau_{\mathrm{pl}}$ для плазмонов в том же диапазоне температур до $200 \mathrm{~K}$ ощутимо выше - от $10^{9}$ до $10^{13} \mathrm{~s}^{-1}$. Более того, сравнение данных [29] с расчетными значениями при низких и высоких температурах показывает, что в области выше $250 \mathrm{~K}$ скорость распада плазмонов заметно превосходит скорость распада экситонов, тогда как в низкотемпературной области $5-15 \mathrm{~K}$ соотношение этих скоростей меняется на обратное. Если предположить, что и в $2 d$-слое $J$-агрегатов цианиновых красителей имеет место аналогичная температурная зависимость для скоростей распада экситонов, то равенство времен $\tau_{\mathrm{pl}}=\tau_{\text {exc }}$ может быть выполнено в некотором интервале области пониженных температур. По нашим оценкам пересечение кривых температурных зависимостей скоростей распада экситонов и плазмонов может происходить в интервале от 10 до $50 \mathrm{~K}$. Таким образом, результаты расчетов динамики энергообмена в плазмонэкситонной системе, полученные на основе упрощенной модели (7)-(9), предполагающей равенство времен $\tau_{\mathrm{pl}}=\tau_{\text {ехс }}$, будут верно отражать картину временных изменений населенностей квазичастиц в температурной области 5-15K. Кинетика распада суммарного числа возбуждений становится экспоненциальной в таком режиме. В других случаях существенного различия времен $\tau_{\mathrm{pl}}, \tau_{\mathrm{exc}}$ кривая общей населенности возбуждений не экспоненциальна, а промодулирована с частотой Раби.

Из рис. 2 и 3 следует, что с уменьшением времени поперечной релаксации $T_{2}$ от $0.8 \tau_{\text {exc }}$ до $0.05 \tau_{\text {exс }}$ при частоте Раби $\Omega=(15-20) \cdot 10^{12} \mathrm{~s}^{-1}$ наблюдается уменьшение глубины модуляции населенностей $\rho_{11}(t)$ и $\rho_{22}(t)$ вплоть до ее практически полного исчезновения. С возрастанием частоты $\Omega$ до значения $30 \cdot 10^{12} \mathrm{~s}^{-1}$ модуляция $\rho_{11}(t)$ и $\rho_{22}(t)$ становится вновь отчетливо выраженной.

В более общей модели (16)-(22) „размороженными“ остаются все три релаксационных параметра $\tau_{\mathrm{pl}}, \tau_{\mathrm{exc}}$, $T_{2}$ и учитывается возможность отстройки от резонанса: $\Delta E \neq 0$. На рис. 5 и 6 представлены временные зависимости населенностей плазмонной и экситонной подсистем, рассчитанные на основе модели (16)-(22). При существенных различиях (вплоть до порядка величины и выше) собственных времен жизни экситонов и плазмонов $\tau_{\mathrm{exc}}, \tau_{\mathrm{pl}}$ наблюдаются характерные модуляции с частотой Раби $\Omega$ не только парциальных плотностей $\rho_{11}(t)$

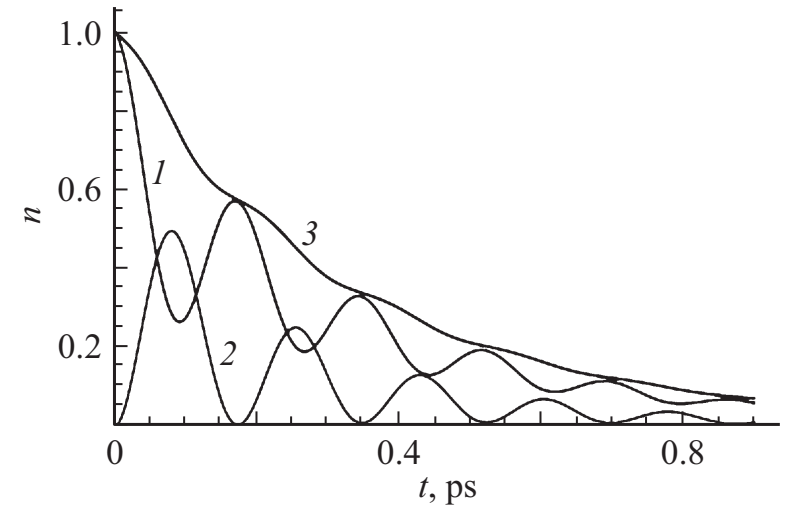

Рис. 5. Кинетика населенностей плазмонной $\rho_{11}(t)(1)$ и экситонной $\rho_{22}(t)(2)$ подсистем, а также суммарной населенности $n(t)=\rho_{11}+\rho_{22}(3)$ возбужденного состояния системы при различных скоростях распада $\tilde{k}_{1}=5.5 T_{2}^{-1}, \tilde{k}_{2}=0.6 T_{2}^{-1}$, времени поперечной релаксации $T_{2}=1 \mathrm{ps}$ и частоте Раби $\Omega=30 T_{2}^{-1}$. Время $t$ выражено в рs. Отстройка от резонанса $\Delta E / \hbar=20 T_{2}^{-1}$.

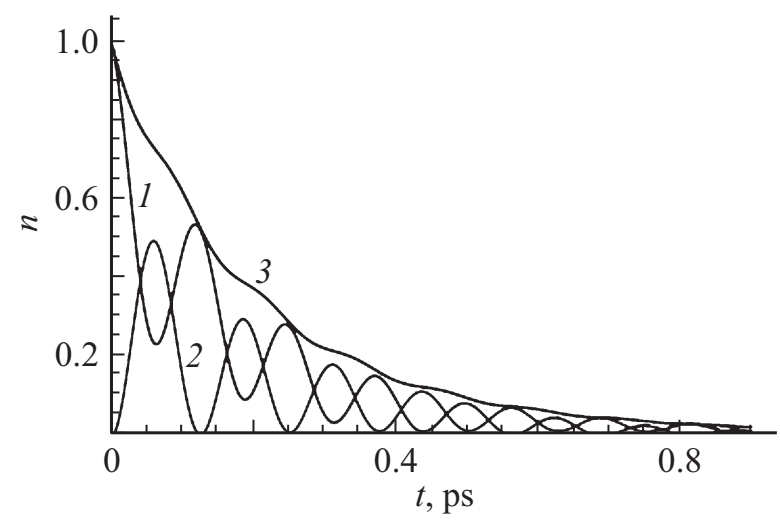

Рис. 6. Кинетика населенностей плазмонной $=\rho_{11}(t)(1)$ и экситонной $\rho_{22}(t)(2)$ подсистем, а также суммарной населенности $n(t)=\rho_{11}+\rho_{22}$ (3) возбужденного состояния системы при различных добавочных скоростях распада $\tilde{k}_{1}=-5.510^{12}, \tilde{k}_{2}=-0.03 \cdot 10^{12} \mathrm{~s}^{-1}$, скорости поперечной релаксации $T_{2}^{-1}=7 \cdot 10^{12} \mathrm{~s}^{-1}$ и частоте Раби $\Omega=40 \cdot 10^{12} \mathrm{~s}^{-1}$. Отстройка от резонанса $\Delta E / \hbar=30 \cdot 10^{12} \mathrm{~s}^{-1}$.

и $\rho_{22}(t)$ квазичастиц, но и суммарной плотности электронных возбуждений $n(t)=\rho_{11}(t)+\rho_{22}(t)$, с заметными ее отклонениями от трендовой экспоненты (рис. 5, 6). Увеличение частоты Раби от $20 \cdot 10^{12}$ до $80 \cdot 10^{12} \mathrm{~s}^{-1}$ приводит к существенному росту глубины модуляции плотности $\rho_{11}(t)$ плазмонов при соотношении скоростей релаксации $\tilde{k}_{1}=5.5 T_{2}^{-1}, \tilde{k}_{2}=0.1 T_{2}^{-1}$ (рис. 7,a). При этом модуляция плотности $\rho_{22}(t)$ экситонов на частоте Раби осуществляется практически до нулевых значений за время $t \sim \Omega^{-1}$.

Представляет интерес случай начальной активации смешанного состояния гибрида $\widehat{\rho}(0)=\left[c_{1}|1\rangle\langle 1|+\right.$ $\left.+c_{2}|2\rangle\langle 2|\right], c_{1}+c_{2}=1$. Тогда временная зависимость парциальной населенности экситонного состояния 

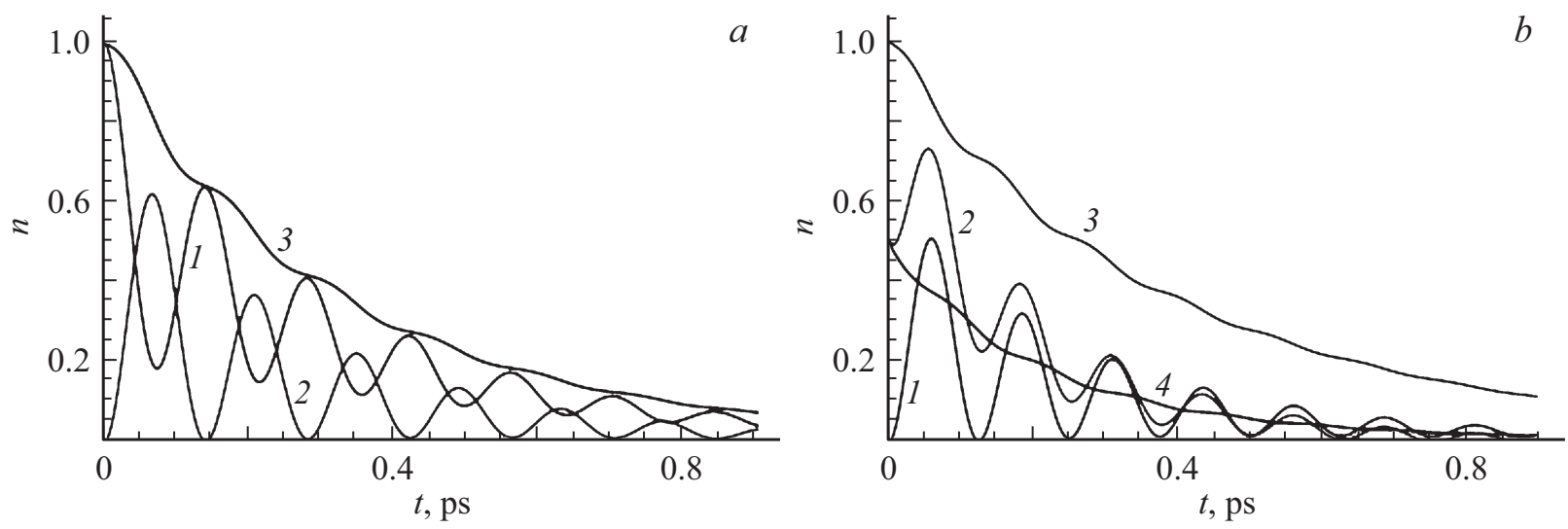

Рис. 7. Кинетика парциальных населенностей возбужденных состояний экситон-плазмонной системы при различных добавочных скоростях распада $\tilde{k}_{1}=5.5 T_{2}^{-1}, \tilde{k}_{2}=0.1 T_{2}^{-1}$ этих состояний и начальных условиях, $T_{2}=1 \mathrm{ps}, \Omega=40 / T_{2}$, время $t$ выражено в ps: $(a)$ кинетики плазмонной $\rho_{11}(t)(1)$ и экситонной $\rho_{2}(t)(2)$ подсистем, а также суммарной населенности $n(t)=\rho_{11}+\rho_{22}(3)$, $\Delta E / h=20 / T_{2} ;(b)$ кинетики экситонной $\rho_{22}(t)$ подсистемы при стартовом заселении $\rho_{11}(0)=1$ только парциального плазмонного состояния (1), чистого начального состояния (26) равноамплитудной суперпозиции (2), смешанного начального состояния (24) с одинаковыми весовыми коэффициентами (4), а также суммарная населенность $n(t)=\rho_{11}+\rho_{22}$ экситон-плазмонной системы $(3)$.

такова:

$$
\begin{aligned}
& \langle 2|\widehat{\rho}(t)| 2\rangle_{\operatorname{mix}}=\exp \left(-t / T_{2}\right)\left[\frac{\exp \left(\kappa_{1}^{(-)} t\right) \exp \left(\kappa_{1}^{(+)} t\right)}{\left(\kappa_{1}^{(-)}-\kappa_{2}^{(-)}\right)\left(\kappa_{1}^{(+)}-\kappa_{2}^{(+)}\right)}\right. \\
& \times\langle 2|\left(\widehat{K}^{(-)}-\kappa_{1}^{(-)} \widehat{I}\right)\left[c_{1}|1\rangle\left\langle 1\left|+c_{2}\right| 2\right\rangle\langle 2|\right] \\
& \times\left(\widehat{K}^{(+)}-\kappa_{1}^{(+)} \widehat{I}\right)|2\rangle+\frac{\exp \left(\kappa_{2}^{(-)} t\right) \exp \left(\kappa_{2}^{(+)} t\right)}{\left(\kappa_{2}^{(-)}-\kappa_{1}^{(-)}\right)\left(\kappa_{2}^{(+)}-\kappa_{1}^{(+)}\right)} \\
& \times\langle 2|\left(\widehat{K}^{(-)}-\kappa_{2}^{(-)} \widehat{I}\right)\left[c_{1}|1\rangle\left\langle 1\left|+c_{2}\right| 2\right\rangle\langle 2|\right] \\
& \times\left(\widehat{K}^{(+)}-\kappa_{2}^{(+)} \widehat{I}\right)|2\rangle+\frac{\exp \left(\kappa_{1}^{(-)} t\right) \exp \left(\kappa_{2}^{(+)} t\right)}{\left(\kappa_{1}^{(-)}-\kappa_{2}^{(-)}\right)\left(\kappa_{2}^{(+)}-\kappa_{1}^{(+)}\right)} \\
& \times\langle 2|\left(\widehat{K}^{(-)}-\kappa_{1}^{(-)} \widehat{I}\right)\left[c_{1}|1\rangle\left\langle 1\left|+c_{2}\right| 2\right\rangle\langle 2|\right] \\
& \times\left(\widehat{K}^{(+)}-\kappa_{2}^{(+)} \widehat{I}\right)|2\rangle+\frac{\exp \left(\kappa_{2}^{(-)} t\right) \exp \left(\kappa_{1}^{(+)} t\right)}{\left(\kappa_{2}^{(-)}-\kappa_{1}^{(-)}\right)\left(\kappa_{1}^{(+)}-\kappa_{2}^{(+)}\right)} \\
& \times\langle 2|\left(\widehat{K}^{(-)}-\kappa_{2}^{(-)} \widehat{I}\right)\left[c_{1}|1\rangle\left\langle 1\left|+c_{2}\right| 2\right\rangle\langle 2|\right] \\
& \left.\times\left(\widehat{K}^{(+)}-\kappa_{1}^{(+)} \widehat{I}\right)|2\rangle\right]
\end{aligned}
$$

Для смешанного начального состояния с равными весовыми коэффициентами экситонной и плазмонной компонент, т. е. когда $c_{1}=c_{2}=1 / 2$, из (23) получаем

$$
\widehat{\rho}(0)=\frac{1}{2}[|1\rangle\langle 1|+| 2\rangle\langle 2|] \text {. }
$$

В этом случае равновесовой начальной смеси кинетика парциальной населенности $\rho_{22}(t)$ определяется выражением

$$
\begin{aligned}
& \langle 2|\widehat{\rho}(t)| 2\rangle_{\operatorname{mix}}=\exp \left(-t / T_{2}\right) \\
& \times \frac{1}{2}\left[\frac{\exp \left(\kappa_{1}^{(-)} t\right) \exp \left(\kappa_{1}^{(+)} t\right)}{\left(\kappa_{1}^{(-)}-\kappa_{2}^{(-)}\right)\left(\kappa_{1}^{(+)}-\kappa_{2}^{(+)}\right)}\right. \\
& \times\left[K_{21}^{(-)} K_{12}^{(+)}+\left(K_{22}^{(-)}-\kappa_{1}^{(-)}\right)\left(K_{22}^{(+)}-\kappa_{1}^{(+)}\right)\right] \\
& +\frac{\exp \left(\kappa_{2}^{(-)} t\right) \exp \left(\kappa_{2}^{(+)} t\right)}{\left(\kappa_{2}^{(-)}-\kappa_{1}^{(-)}\right)\left(\kappa_{2}^{(+)}-\kappa_{1}^{(+)}\right)} \\
& \times\left[K_{21}^{(-)} K_{12}^{(+)}+\left(K_{22}^{(-)}-\kappa_{2}^{(-)}\right)\left(K_{22}^{(+)}-\kappa_{2}^{(+)}\right)\right] \\
& +\frac{\exp \left(\kappa_{1}^{(-)} t\right) \exp \left(\kappa_{2}^{(+)} t\right)}{\left(\kappa_{1}^{(-)}-\kappa_{2}^{(-)}\right)\left(\kappa_{2}^{(+)}-\kappa_{1}^{(+)}\right)} \\
& \times\left[K_{21}^{(-)} K_{12}^{(+)}+\left(K_{22}^{(-)}-\kappa_{1}^{(-)}\right)\left(K_{22}^{(+)}-\kappa_{2}^{(+)}\right)\right] \\
& \left.\times\left[K_{21}^{(-)} K_{12}^{(+)}+\left(K_{22}^{(-)}-\kappa_{2}^{(-)}\right)\left(K_{22}^{(+)}-\kappa_{1}^{(+)}\right)\right]\right] \\
& \left.+\kappa_{2}^{(-)}-\kappa_{1}^{(-)}\right)\left(\kappa_{1}^{(+)}-\kappa_{2}^{(+)}\right)
\end{aligned}
$$


График функции $\rho_{22}(t)$, определенной выражением (25), представлен на рис. 7, $b$ кривой 4. Мы видим, что для такого случая приготовленного начального состояния осцилляции населенности парциального экситонного состояния не являются отчетливо выраженными.

Кроме рассмотренного смешанного случая начальное состояние системы может быть чистым, но суперпозиционным, т.е. составленным из чистых состояний $|1\rangle$ и $|2\rangle:| \pm\rangle=\alpha|1\rangle \pm \beta|2\rangle$. Тогда начальное состояние оператора плотности, отвечающее чистому состоянию $|+\rangle=\alpha|1\rangle+\beta|2\rangle$, таково:

$$
\widehat{\rho}(0)=|+\rangle\langle+|=\left[(\alpha|1\rangle+\beta|2\rangle)\left(\alpha^{*}\langle 1|+\beta^{*}\langle 2|\right)\right]
$$

или

$$
\begin{aligned}
\widehat{\rho} & =|+\rangle\langle+|=\left[|\alpha|^{2}|1\rangle\left\langle\left. 1|+| \beta\right|^{2} \mid 2\right\rangle\langle 2|\right. \\
& +\alpha \beta^{*} \mid 1\left\langle\left\langle 2\left|+\alpha^{*} \beta\right| 2\langle\langle 1|] .\right.\right.
\end{aligned}
$$

В области пересечения дисперсионных кривых (рис. 1) взаимодействие $V_{12}$ становится относительно большим, а коэффициенты $\alpha, \beta$ в суперпозиции - равными: $\alpha \approx \beta \approx 1 / \sqrt{2}$ :

$$
\widehat{\rho}(0)=|+\rangle\langle+|=\frac{1}{2}[|1\rangle\langle 1|+| \mid 2\rangle\langle 2|+| 1\rangle\langle 2|+| 2\rangle\langle 1|] .
$$

Сравнивая этот случай чистого начального состояния с равновесовой смесью (24), замечаем, что в $\rho_{22}(t)$ добавляются члены, которые можно объединить в отдельную группу:

$$
\begin{aligned}
& \langle 2 \mid \widehat{\rho}(t) 2\rangle_{\operatorname{edd}}=\exp \left(-t / T_{2}\right) \\
& \times \frac{1}{2}\left[\frac{\exp \left(\kappa_{1}^{(-)} t\right) \exp \left(\kappa_{1}^{(+)} t\right)}{\left(\kappa_{1}^{(-)}-\kappa_{2}^{(-)}\right)\left(\kappa_{1}^{(+)}-\kappa_{2}^{(+)}\right)}\right. \\
& \times\left[K_{21}^{(-)}\left(K_{22}^{(+)}-\kappa_{1}^{(+)}\right)\left(K_{22}^{(-)}-\kappa_{1}^{(-)}\right) K_{12}^{(+)}\right] \\
& +\frac{\exp \left(\kappa_{2}^{(-)} t\right) \exp \left(\kappa_{2}^{(+)} t\right)}{\left(\kappa_{2}^{(-)}-\kappa_{1}^{(-)}\right)\left(\kappa_{2}^{(+)}-\kappa_{1}^{(+)}\right)} \\
& \times\left[K_{21}^{(-)}\left(K_{22}^{(+)}-\kappa_{2}^{(+)}\right)+\left(K_{22}^{(-)}-\kappa_{2}^{(-)}\right) K_{12}^{(+)}\right] \\
& +\frac{\exp \left(\kappa_{1}^{(-)} t\right) \exp \left(\kappa_{2}^{(+)} t\right)}{\left(\kappa_{1}^{(-)}-\kappa_{2}^{(-)}\right)\left(\kappa_{2}^{(+)}-\kappa_{1}^{(+)}\right)} \\
& \times\left[K_{21}^{(-)}\left(K_{22}^{(+)}-\kappa_{2}^{(+)}\right)+\left(K_{22}^{(-)}-\kappa_{1}^{(-)}\right) K_{12}^{(+)}\right] \\
& \left.\times\left[K_{21}^{(-)}\left(K_{22}^{(+)}-\kappa_{1}^{(+)}\right)+\left(K_{22}^{(-)}-\kappa_{2}^{(-)}\right) K_{12}^{(+)}\right]\right] . \\
& +\frac{\exp \left(\kappa_{2}^{(-)} t\right) \exp \left(\kappa_{1}^{(+)} t\right)}{\left(\kappa_{2}^{(-)}-\kappa_{1}^{(-)}\right)\left(\kappa_{1}^{(+)}-\kappa_{2}^{(+)}\right)}
\end{aligned}
$$

Таким образом, для случая суперпозиционного чистого начального состояния кинетика населенности $\rho_{22}(t)$ представляет собой сумму двух компонент

$$
\langle 2|\widehat{\rho}(t)| 2\rangle_{\text {pure }}=\langle 2|\widehat{\rho}(t)| 2\rangle_{\text {mix }}+\langle 2|\widehat{\rho}(t)| 2\rangle_{\text {add }} .
$$

В результате перегруппировки членов получаем

$$
\begin{aligned}
& \langle 2 \mid \widehat{\rho}(t) 2\rangle_{\text {pure }}=\frac{1}{2} \exp \left(-t / T_{2}\right) \\
& \times\left[\frac{\exp \left(\kappa_{1}^{(-)} t\right) \exp \left(\kappa_{1}^{(+)} t\right)}{\left(\kappa_{1}^{(-)}-\kappa_{2}^{(-)}\right)\left(\kappa_{1}^{(+)}-\kappa_{2}^{(+)}\right)}\right. \\
& \times\left(K_{22}^{(+)}+K_{12}^{(+)}-\kappa_{1}^{(+)}\right)\left(K_{21}^{(-)}+K_{22}^{(-)}-\kappa_{1}^{(-)}\right) \\
& +\frac{\exp \left(\kappa_{2}^{(-)} t\right) \exp \left(\kappa_{2}^{(+)} t\right)}{\left(\kappa_{2}^{(-)}-\kappa_{1}^{(-)}\right)\left(\kappa_{2}^{(+)}-\kappa_{1}^{(+)}\right)} \\
& \times\left(K_{22}^{(+)}+K_{12}^{(+)}-\kappa_{2}^{(+)}\left(K_{21}^{(-)}+K_{22}^{(-)}-\kappa_{2}^{(-)}\right)\right. \\
& +\frac{\exp \left(\kappa_{1}^{(-)} t\right) \exp \left(\kappa_{2}^{(+)} t\right)}{\left(\kappa_{1}^{(-)}-\kappa_{2}^{(-)}\right)\left(\kappa_{2}^{(+)}-\kappa_{1}^{(+)}\right)} \\
& \times\left(K_{22}^{(+)}+K_{12}^{(+)}-\kappa_{2}^{(+)}\right)\left(K_{21}^{(-)}+K_{22}^{(-)}-\kappa_{1}^{(-)}\right) \\
& +\frac{\exp \left(\kappa_{2}^{(-)} t\right) \exp \left(\kappa_{1}^{(+)} t\right)}{\left(\kappa_{2}^{(-)}-\kappa_{1}^{(-)}\right)\left(\kappa_{1}^{(+)}-\kappa_{2}^{(+)}\right)}
\end{aligned}
$$

График этой функции представлен на рис. 7, $b$ кривой 2. Сравнивая его с кривой 4 этого же рисунка, заключаем, что за глубоко модулированные осцилляции кривой 2 отвечает группа слагаемых (27).

В альтернативном подходе решения кинетической задачи, следуя Бурштейну и Конышеву [27], исходную систему уравнений (4) для элементов матрицы плотности можно подвергнуть преобразованию Лапласа с целью исключения временных зависимостей матричных элементов:

$$
L\left[\rho_{i j}\right]=\int_{0}^{\infty} \rho_{i j}(t) \exp (-s t) d t, \quad L\left[\dot{\rho}_{i j}\right]=s L\left[\rho_{i j}\right]-\rho_{i j}(0)
$$

Тогда величина

$$
1 / \tau_{1}^{\text {ef }}=\left(\int_{0}^{\infty} \rho_{11}(t) \exp (-s t) d t\right)_{s=0}^{-1}=1 / L\left[\rho_{11}\right]_{s=0}
$$

может рассматриваться как обобщенная скорость распада населенности в донорной подсистеме, в том числе - 
за счет многоактного переноса энергии к акцептору, за все время существования активированной системы. В [27] для нее получено выражение:

$$
\frac{1}{\tau_{1}^{\mathrm{ef}}}=\frac{1}{L\left[\rho_{11}\right]_{s=0}}=\frac{1}{\tau_{1}}+\frac{2\left|V_{12}\right|^{2} T_{2} / \hbar^{2}}{1+\left(\frac{T_{2} \Delta E}{\hbar}\right)^{2}+2 \frac{\left|V_{12}\right|^{2}}{\hbar^{2}} T_{2} \tau_{2}} .
$$

При использовании (30) осцилляции населенности в ходе энергообмена в системе игнорируются, но в отличие от теории Ферстера формула (30) может быть использована при произвольном значении экситон-плазмонного взаимодействия $V_{12}$. Сглаженная (экспоненциальная) кинетика эффективного распада населенности донорной подсистемы при таком подходе определяется уравнением $\dot{n}_{\mathrm{D}}(t)=-n_{\mathrm{D}}(t) / \tau_{1}^{\text {ef }}$. В то же время очевидно, что, несмотря на привлекательность метода (29), (30), связанную с его простотой и наглядностью, введения эффективной скорости $1 / \tau_{1}^{\text {ef }}$ релаксации донорной подсистемы может оказаться недостаточно для описания кинетики процессов, требующего детализированных данных высокого временного разрешения.

Дополнительного обсуждения также требует принятие в моделях (4), (5) и (16) приближения марковской релаксации. В этих моделях используются константы релаксации, определенные для не взаимодействующих плазмонной и экситонной систем. В то же время рассматриваемые величины экситон-плазмонного взаимодействия превышают эти константы релаксации в десятки раз, что может приводить к существенной перестройке спектра гибридной системы (1). Может показаться, что вследствие такой перестройки будут изменяться релаксационные параметры. Однако при сильном экситонплазмонном взаимодействии использование в работе приближения марковской релаксации является не менее обоснованным, чем при слабой экситон-плазмонной связи, так как в этом случае характерные частоты системы существенно превышают скорости релаксации, которые могут считаться не зависящими от времени величинами.

Скорости релаксации определяются характерными константами взаимодействия рассматриваемых подсистем (экситонной и электронной) с термостатом. Если эти константы достаточно малы, то даже сильное экситон-плазмонное взаимодействие, видоизменяющее спектр объединенной системы по сравнению со спектрами не взаимодействующих компонентов, не будет приводить к существенным изменениям времен релаксации кооператива. По этой причине в настоящей работе мы полагаем скорости релаксации экситонной и плазмонной подсистем неизменными после включения взаимодействия между ними.

В системах с большим числом свободы сложные внутренние движения могут считаться тепловыми, и система может рассматриваться в качестве термостата. Фазовое пространство динамической подсистемы (экситоны и плазмоны) полагается достаточно малым, а взаимодействие ее с термостатом слабым. Тогда гамильтониан этого взаимодействия практически не изменяет характерные частоты движения подсистемы. В рассматриваемом нами случае термостатом является фононный резервуар подложки и диэлектрических слоев сэндвичевой наноструктуры. В случае малых времен корреляции флуктуационных движений термостата формируется невысокая постоянная скорость релаксации, и уравнение для оператора плотности экситон-плазмонной системы может быть записано в марковском приближении с теми же временами релаксации, что были в отсутствие экситон-плазмонного взаимодействия.

Другой разновидностью термостата в рассматриваемой системе является фотонный резервуар. Релаксация системы при взаимодействии с ним осуществляется в виде спонтанного испускания фотона экситонной подсистемой, что дает соответствующий вклад в общую скорость продольной релаксации $1 / T_{1}$. Как и в случае фононного термостата, мы полагали эту скорость практически не изменившейся после „включения“ экситон-плазмонного взаимодействия.

Скорость релаксационных переходов при слабом взаимодействии динамической системы с термостатом определяется двумя факторами: собственно величиной этого взаимодействия и спектральной плотностью шума термостата на частотах динамической системы. Если в диапазоне частот перестроенного спектра системы (существенно измененного из-за сильного экситонплазмонного взаимодействия) спектральная плотность шума термостата имеет пологий участок (локально „белый“ диапазон спектра), то, несмотря на перестроенный спектр экситон-плазмонной системы, скорости релаксации ее компонентов могут не изменяться существенным образом.

Однако даже в случае заметного изменения парциальных скоростей релаксации системы, произошедшего из-за резонанса с модами термостата, это обстоятельство, на наш взгляд, не повлияет на главные результаты работы. Посредством специально разработанной математической процедуры были получены простые и удобные для анализа выражения для собственных значений кинетического оператора системы в термостате. Из этих выражений легко прослеживается переключение на режим осцилляций Раби с произвольно большим значением частоты безотносительно к возможному изменению парциальных скоростей релаксации. Таким образом, итоговые выражения работы и сделанные на их основе выводы не будут изменяться даже в ситуации, когда могут возникать резонансы сильно перестроенных частот объединенной системы с хорошо выраженными в спектре термостата модами.

Учет эффектов немарковости не был осуществлен здесь, поскольку он представляет собой отдельную, достаточно сложную задачу с акцентами на других особенностях рассмотренной системы.

Таким образом, в настоящей работе на основе специальной модели планарной плазмон-экситонной системы 
установлена возможность появления осцилляционного режима в кинетике распада-активации (энергообмена) компонентов гибрида при сильном их взаимодействии друг с другом и термостатом. Кроме того, в работе были рассмотрены случаи чистых и смешанных начальных состояний экситон-плазмонной системы, различным образом проявляющиеся в кинетике ее распада, не всегда демонстрирующей наличие осцилляционного режима. Обнаруженные особенности временного поведения разносортной системы квазичастиц могут быть полезными при анализе работы фотоэлектронных устройств с реализованным в них сильным плазмон-экситонным взаимодействием в субнаносекундной временной области. Важным может оказаться не только сам факт одностороннего результирующего переноса энергии между компонентами системы, но и немонотонный временной характер такого процесса. Изменяя толщину изолирующей прослойки между двумерными экситонами и поверхностными плазмон-поляритонами в планарной наноструктуре, можно осуществлять переключение кинетических режимов энергообмена между ее компонентами, от возвратно-осцилляционного до однонаправленного релаксационного.

Работа выполнена при поддержке грантами РФФИ и правительства Оренбургской области (проект № 16-42-560671), а также по Госзаданию Министерства образования и науки РФ (проект № 233).

\section{Список литературы}

[1] Ambjörnsson T., Mukhopadhyay G., Apell S.P., Käll M. // Phys. Rev. B. 2006. V. 73. P. 085412.

[2] Akselrod G.M., Young E.R., Bradley M.S., Bulovic V. // Opt. Expr. 2013. V. 21. N 10.doi 10.1364/OE.21.012122

[3] Belless J., Symonds C., Laverdant J., Benoit J.-M., Plenet J.C., Vignoli S. // Electronics. 2014. N 3. P. 303.

[4] Витухновский А.Г., Чубич Д.А. Патент РФ № 2417483. 2009.

[5] Лебедев В.С., Медведев А.С., Васильев Д.Н., Чубич Д.А., Витухновский А.Г. // Квант. электрон. 2010. Т. 40. № 3. C. 246.

[6] Balci S., Kocabas C., Ates S., Karademir E., Salihoglu O., Aydinli A. // Phys. Rev. B. 2012. V. 86. P. 235402.

[7] Goliney I.Yu., Sugakov V.I., Valkunas L., Vertsimakha G.V. // Chem. Phys. 2012. V. 404. P. 116.

[8] Gonzalez-Tudela A., Huidobro P.A., Martin-Moreno L., Tejedor C., Garcia-Vidal F.J. // Phys. Rev. Lett. 2013. V. 110. P. 126801.

[9] Gonzalez-Ballestero C., Feist J., Moreno E., GarciaVidal F.J. // Phys. Rev. B. 2015. V. 92. P. 121402.

[10] Strokova Y.A., Saletsky A.M., Kucherenko M.G. // RussianJapanese Conference „Chemical Physics of Molecules and Polyfunctional Materials“: Proceedings. 29-31 October 2014. OSU, Orenburg, Russia / IPK „Universitet“, 2014. P. 16.

[11] Кособукин В.A. // ФТТ. 2015. Т. 57. В. 7. С. 1413.

[12] Agranovich V.M., Gartstein Yu.N., Litinskaya M. // Chem. Rev. 2011. V. 111. P. 5179.
[13] Agranovich V.M., Basko D.M., La Rocca G.C. // Phys. Rev. B. 2012. V. 86. P. 165204.

[14] Agranovich V.M., Dubovskii O.A., La Rocca G.C. // Pis'ma v ZhETF. 2014. V. 99. N 6. P. 371.

[15] Кучеренко М.Г., Чмерева Т.М. // ФТТ. 2008. Т. 50. №3. C. 512.

[16] Strokova Y.A., Kucherenko M.G. // J. Phys/: Conf/ Ser. 2014. V. 541. N 1. Article number 012088.

[17] Чмерева Т.М., Кучеренко М.Г., Дмитриев А.Д. // Опт. и спектр. 2015. T. 118. № 2. С. 300.

[18] Klimov V.V., Letokhov V.S. // Phys. Rev. A. 1998. V. 58. N 4. P. 3235.

[19] Кучеренко М.Г., Степанов В.Н., Кручинин Н.Ю. // Опт. и спектр. 2015. T. 118. № 1. С. 107.

[20] Кучеренко М.Г., Чмерева Т.М. // Вестник ОГУ. 2015. № 9 (184). C. 45.

[21] Чмерева Т.М., Кучеренко М.Г., Курмангалеев К.С. // Опт. и спектр. 2016. Т. 120. № 6. С. 941.

[22] Давыдов А.С. Квантовая механика. Наука, 1973. 704 с.

[23] Davydov A.S., Serikov A.A. // Phys. Stat. Sol. (B). 1972. V. 51. N 1. P. 57.

[24] Агранович В.М., Галанин М.Д. Перенос энергии электронного возбуждения в конденсированных средах. М.: Наука, 1978. $384 \mathrm{c}$.

[25] Блум K. Теория матрицы плотности и ее приложения. М.: Мир, 1983. 248 с.

[26] Беллман Р. Введение в теорию матриц. М.: Наука, 1976. $352 \mathrm{c}$.

[27] Конышев В.П., Бурштейн А.И. // Теор. экспер. химия. 1968. T. 4. C. 192.

[28] Johnson R.C., Merrifield R.E. // Phys. Rev. B. 1970. V. 1. N 2. P. 896.

[29] Бродин М.С. Поляритоны в кристаллооптике. Киев: Наукова Думка, 1984. 200 с. 